\title{
Observational properties of galaxies in small systems
}

\author{
M. Jõeveer \\ Tartu Observatory, Tartumaa 61602, Estonia
}

\begin{abstract}
.
The mean absolute luminosities and diameters of galaxies in double and triple configurations are compared with the same characteristics for single galaxies. Members of optical pairs and triplets are used as the sample of single galaxies. The mean luminosities of galaxies in physical pairs and triplets are found to be the same as for single galaxies. At the same absolute luminosity, single galaxies have larger diameters compared to galaxies in pairs and triplets.
\end{abstract}

\section{Introduction}

The Hubble Deep Field project has shown that among distant galaxies there is an intriguingly high proportion of anomalous forms. There are apparently interacting pairs with multiple structure, galaxies with superluminous star-forming regions, low surface brightness galaxies of various forms and also compact galaxies. The problem is how they have evolved into the galaxies of contemporary forms around us.

It is highly probable that interactions followed by merging play an active role in the evolution of galaxies. Through the merging process, the main characteristics of galaxies, their absolute luminosities and diameters must have changed. It is natural to suppose that more massive galaxies have suffered more mergers. Thus, by means of comparison of mean observational properties of galaxies in different environments some information about their evolutionary history might be obtained.

According to previous studies (White and Valders 1980; Karachentsev 1987, 1990) galaxies in binary systems are $1.7-2.2$ times brighter than single galaxies. This excess of the mean luminosity was interpreted by Wiren et al. (1996) as evidence that binary galaxies have suffered more mergers than single galaxies.

If we compare the characteristics of galaxies in systems with those of single galaxies, the role of the selection criteria of the samples is decisive. In the present study the properties of galaxies in binary and triple systems are compared with properties of galaxies in optical binaries and triplets, which are treated as representatives of single galaxies, selected on the basis of the same criteria as the galaxies in systems. Systems with mass-to-luminosity ratios $M / L<100$ in solar units are considered as physical systems, others as optical or in most cases optical systems. 


\section{Galaxies in isolated pairs}

The largest sample of binary galaxies compiled on the basis of well-defined criteria is the "Catalogue of isolated pairs of galaxies in northern hemisphere" (Karachentsev 1972). Mean luminosities $M$ in the Holmberg system and diameters $A_{25}$ are shown in Table 1 on the basis of the observational data published in Karachentsev's monograph "Binary Galaxies" (Karachentsev 1987). We see that the galaxies in optical pairs (i.e. single galaxies) have practically the same mean luminosities as the galaxies in physical pairs.

The strong similarity of mean luminosities and diameters of the galaxies in physical and optical pairs arouses some suspicion that the division of the pairs into optical and physical systems according to the formal $M / L$ criterion is not quite right. Of course, the data on interactions and proportions of the Markarian galaxies (Table 2) convince us that the $M / L$ criterion works. Significantly higher proportions of interactions and Markarian galaxies clearly advocate for $M / L<$ 100 pairs being physical. However, at the same time, data from Table 2 indicate that nearly half of the pairs with $100<M / L<300$ and even some pairs among $300<M / L<1000$ sample may be physical systems.

\section{Galaxies in isolated triplets of galaxies}

In Table 3 properties of the galaxies in physical and optical triplets are presented. These come from the catalogue of 84 isolated triplets of galaxies in the northern hemisphere by Karachentseva et al. (1979). The division into the physical and optical systems is the same as in the paper by Karachentsev et al. (1989). Absolute luminosities $\mathrm{M}$ in the Holmberg system and diameters $A_{25}\left(H_{0}=75\right.$ $\mathrm{km} \mathrm{s}^{-1} \mathrm{Mpc}^{-1}$ ) for individual galaxies have been computed on the basis of the data in the paper by Karachentseva et al. (1988).

As in the case of binary systems, we see that differences between the properties of triple, double and single galaxies are small. Mean luminosities of single galaxies are even higher in comparison with galaxies in the systems, but on the basis of this small sample it is quite risky to draw some definite conclusions. It seems that at the same luminosity single galaxies are slightly larger (of lower surface brightness) in comparison with galaxies in physical triplets.

\section{Conclusions}

- Considering member galaxies of optical pairs and triplets as a representative sample of single galaxies, mean luminosities of galaxies in physical pairs and triplets are the same as for single galaxies.

- At the same absolute luminosity single galaxies have larger diameters $A_{25}$ (they are of lower surface brightness) in comparison with the galaxies in physical pairs and triplets.

- Galaxies in systems have more interactions and their mass-to-luminosity ratios are lower when compared to non-interactive galaxies. Hence, it is possible that the mean masses of the galaxies in systems are lower in comparison with the masses of single galaxies and single galaxies, not the galaxies in small systems, have suffered more mergers. 
Table 1. Mean absolute luminosities and diameters of member galaxies of isolated pairs of galaxies

\begin{tabular}{l|c|ccc|c}
\hline & $\begin{array}{c}\text { Number } \\
\text { of pairs }\end{array}$ & $\begin{array}{c}\text { Brighter } \\
\text { components }\end{array}$ & $\begin{array}{c}\text { Fainter } \\
\text { components }\end{array}$ & Both & $\mathrm{kpc}$ \\
\hline $\begin{array}{l}M / L<100 \\
\text { (phys. systems) }\end{array}$ & 487 & $-20.54 \pm 0.06$ & $-19.95 \pm 0.06$ & $-20.23 \pm 0.04$ & $23.6 \pm 0.8$ \\
\hline $100<M / L<300$ & 28 & $-20.57 \pm 0.22$ & $-20.05 \pm 0.22$ & $-20.31 \pm 0.18$ & $28.4 \pm 3.2$ \\
\hline $\begin{array}{l}300<M / L<1000 \\
\text { (opt. systems) }\end{array}$ & 16 & $-20.59 \pm 0.30$ & $-19.87 \pm 0.30$ & $-20.23 \pm 0.24$ & $28.1 \pm 4.2$ \\
\hline $\begin{array}{l}1000<M / L \\
\text { (opt. systems) }\end{array}$ & 54 & $-20.79 \pm 0.17$ & $-19.47 \pm 0.17$ & $-20.13 \pm 0.13$ & $27.0 \pm 2.2$ \\
\hline $\begin{array}{l}300<M / L \\
\text { (opt. systems) }\end{array}$ & 70 & $-20.74 \pm 0.16$ & $-19.56 \pm 0.16$ & $-20.15 \pm 0.11$ & $27.3 \pm 2.1$ \\
\hline
\end{tabular}

Table 2. Interactions in isolated pairs of galaxies

\begin{tabular}{lrrrrrr}
\hline Sample of pairs & $\begin{array}{r}\text { Number } \\
\text { of pairs }\end{array}$ & $\begin{array}{r}\text { Number } \\
\text { of inter- } \\
\text { actions }\end{array}$ & $\begin{array}{r}\text { Percentage } \\
\text { of inter- } \\
\text { actions }\end{array}$ & $\begin{array}{r}\text { Percentage } \\
\text { of optical } \\
\text { pairs }\end{array}$ & $\begin{array}{r}\text { Number of } \\
\text { Markarian } \\
\text { galaxies }\end{array}$ & $\begin{array}{r}\text { Percentage } \\
\text { Markarians }\end{array}$ \\
\hline$M / L<100$ & 487 & 287 & 59 & $0:$ & 66 & 13.6 \\
$100<M / L<300$ & 28 & 11 & 39 & $50 ?$ & 2 & 7 \\
$300<M / L<1000$ & 16 & 5 & 31 & $75 ?$ & 1 & 6 \\
$1000<M / L$ & 54 & 12 & 22 & 100 & 2 & 4 \\
$300<M / L$ & 70 & 17 & 24 & 100 & 3 & 4 \\
\hline
\end{tabular}

Table 3. Mean absolute luminosities and mean diameters of member galaxies of isolated triplets of galaxies

\begin{tabular}{lccc}
\hline Sample of triplets & $\begin{array}{c}\text { Number } \\
\text { of member } \\
\text { galaxies }\end{array}$ & $\langle M\rangle$ & $\begin{array}{c}\left\langle A_{25}\right\rangle \\
\mathrm{kpc}\end{array}$ \\
\hline$M / L<100$ (physical triplets) & 141 & $-20.24 \pm 0.13$ & $21.8 \pm 1.8$ \\
\hline $100<M / L<300$ & 27 & $-20.11 \pm 0.30$ & $23.6 \pm 4.1$ \\
\hline $300<M / L$ (optical triplets) & & & \\
a) galaxies in pairs & 44 & $-20.03 \pm 0.23$ & $25.2 \pm 3.2$ \\
b) single galaxies & 37 & $-20.63 \pm 0.25$ & $30.0 \pm 3.5$ \\
c) singles + pairs & 81 & $-20.30 \pm 0.17$ & $27.4 \pm 2.4$ \\
\hline
\end{tabular}


Acknowledgments. I gratefully acknowledge travel support granted by the Academy of Finland. This work was supported by the Estonian Science Foundation, grant no. 2627.

\section{References}

Karachentsev, I. D. 1972, Soobshch. Spets. Astrofiz. Obs., 7, 3.

Karachentsev, I. D. 1987, Binary galaxies, Moscow, Nauka.

Karachentsev, I. D. Karachentseva, V. E., Lebedev, V. S. 1989, Izv. Spets. Astrofiz. Obs. 27, 67.

Karachentsev, I. D. 1990, Proc. IAU Coll. No 124, 3.

Karachentseva, V. E., Karachentsev, I. D., Shcherbanovskij, A. L. 1979, Izv. Spets. Astrofiz. Obs., 11, 3.

Karachentseva, V. E., Karachentsev, I. D., Lebedev, V. S. 1988, Izv. Spets. Astrofiz. Obs., 26, 42.

White, S. D. M., Valders, F. 1980, MNRAS, 190, 55.

Wiren, S., Zheng, J.-Q., Valtonen, M. J., Chernin, A. D. 1996, AJ, 111, 160. 